\title{
PDIA3 regulates trophoblast apoptosis and proliferation in preeclampsia via the MDM2/p53 pathway
}

\author{
Hui-Qin Mol,2, Fu-Ju Tian"1,2, Xiao-Ling Ma ${ }^{1,2}$, Yu-Chen Zhang ${ }^{1,2}$, Cheng-Xi Zhang ${ }^{1,2}$, Wei-Hong Zengl,2, Yan Zhang ${ }^{3}$ \\ and Yi Lin ${ }^{1,21}$ Institute of Shanghai Key Laboratory of Embryo Original Diseases and Shanghai Municipal Key Clinical \\ Specialty Project Affiliated to Shanghai Jiao Tong University School of Medicine, Shanghai, China, ${ }^{2}$ The International \\ Peace Maternity \& Child Health Hospital, Shanghai Jiao Tong University School of Medicine, Shanghai, China and \\ ${ }^{3}$ Department of Obstetrics and Gynecology, Renmin Hospital of Wuhan University, Wuhan, China
}

Correspondence should be addressed to Y Lin; Email: yilinonline@126.com

\begin{abstract}
Protein disulfide isomerase 3 (PDIA3) is a chaperone protein that modulates the folding of newly synthesized glycoproteins, has isomerase and redox activity, and has been implicated in the pathogenesis of many diseases. However, the role of PDIA3 in pregnancy-associated diseases remains largely unknown. Our present study reveals a key role for PDIA3 in the biology of placental trophoblasts from women with preeclampsia (PE). Immunohistochemistry and Western blot analysis revealed that PDIA3 expression was decreased in villous trophoblasts from women with PE compared to normotensive pregnancies. Further, using a Cell Counting Kit-8 assay, flow cytometry, and 5-ethynyl-2'-deoxyuridine (EdU) staining, we found that siRNA-mediated PDIA3 knockdown significantly promoted apoptosis and inhibited proliferation in the HTR8/SVneo cell line, while overexpression of PDIA3 reversed these effects. Furthermore, RNA sequencing and Western blot analysis demonstrated that knockdown of PDIA3 inhibited MDM2 protein expression in HTR8 cells, concurrent with marked elevation of p53 and p21 expression. Conversely, overexpression of PDIA3 had the opposite effects. Immunohistochemistry and Western blot further revealed that MDM2 protein expression was downregulated and p21 was increased in trophoblasts of women with PE compared to women with normotensive pregnancies. Our findings indicate that PDIA3 expression is decreased in the trophoblasts of women with PE, and decreased PDIA3 induces trophoblast apoptosis and represses trophoblast proliferation through regulating the MDM2/p53/p21 pathway.

Reproduction (2020) 160 293-305
\end{abstract}

\section{Introduction}

Preeclampsia (PE) is an idiopathic gestational complication that affects approximately $2-8 \%$ pregnancies worldwide and is classically characterized by new-onset hypertension and proteinuria, usually after 32 weeks of gestation (Duley 2009). Pregnancies with PE usually result in premature delivery and are associated with high risks of liver damage, eclampsia, and fetal growth restriction. Although the precise etiopathogenesis of preeclampsia remain uncertain, it is widely accepted that PE is a placenta-mediated multisystemic disease. Previous studies showed that the regulation of apoptosis in placental trophoblasts is crucial for the survival, differentiation, and implantation of human placenta (Huppertz et al. 2006) (Sharp et al. 2010). Exaggerated apoptosis of trophoblasts (extravillous trophoblasts in early pregnancy and syncytiotrophoblasts in term placenta) are integrally involved in placental dysfunction and correlates with severe gestational diseases, such as intrauterine growth retardation and PE (Smith et al. 1997, DiFederico et al. 1999). In previous studies, we reported that the apoptosis-related protein BCL2 in trophoblasts plays a critical role in the development of PE (Mo et al. 2019). However, the precise involvement of aberrant apoptosis of placental trophoblasts in the pathogenesis of $\mathrm{PE}$ requires further elucidation.

Protein disulfide isomerase 3 (PDIA3; also referred to as ERp57 and GRP58) is a prominent member of the protein disulfide isomerase (PDI) family. Aberrant expression of PDIA3 has been reported to be involved in the pathogenesis of multiple cancers (Yang et al. 2018) (Wang et al. 2017), neurodegenerative diseases (Tohda et al. 2012, Parakh et al. 2018), and respiratory immune disease (Mutze et al. 2015, Chamberlain et al. 2019). PDIA3 localized to the cell surface is a receptor for the active form of 1,25(OH)2D3 (Vitamin D3), which regulates bone formation (Yuan et al. 2017). Nuclear PDIA3 was reported to directly interact with DNA, resulting in the activation and DNA-binding function of various transcription factors and proteins (Grillo et al. 2006, Aureli et al. 2013). PDIA3 exhibits PDI and redox activity and interacts with calnexin and calreticulin, which act as chaperones, to ensure that newly synthesized proteins are correctly folded (Hebert \& Molinari 2007). Furthermore, PDIA3 is essential 
for major histocompatibility complex (MHC) class I antigen presentation (Garbi Natalio et al. 2005). Stefan Sonderegger reported that IL11 repressed extravillous trophoblasts motility followed by downregulation of PDIA3 protein expression (Sonderegger et al. 2011). A bioinformatics analysis revealed that PDIA3 was highly related with PE (Tejera et al. 2012), but this relationship was not thoroughly explored. Therefore, we hypothesized that PDIA3 might mediate the occurrence of PE.

MDM2 targets p53 for ubiquitination via the proteasome, and p53 is at low, often undetectable, concentrations in normal cells. (Ygal Haupt et al. 1997) (Kubbutat et al., 1997) The p53 protein is only activated when cells are stressed or damaged, resulting in the inhibition of cell proliferation or causing apoptosis. p21 Waf1/Cip1 is a major transcriptional target of activated p53 (Bert Vogelstein \& Levine 2000). Moreover, the phosphorylation of p53 is increased after knockdown of PDIA3 (Krynetskaia et al. 2009). Zou H. et al. observed that PDIA3 was positively correlated with $\mathrm{p} 53$ expression in the progression of diffuse glioma (Zou et al. 2018). Furthermore, the MDM2-p53 pathway was reported to be associated with $\mathrm{PE}$, and protein expression of p53 and p21 was significantly increased in villous trophoblasts of pregnancies with PE (Sharp et al. 2014). These reports led us to study the correlation between the MDM2-p53 pathway and PDIA3 in PE.

In the current study, we aimed to elucidate the potential role of PDIA3 in placental trophoblasts during PE. We hypothesized that PDIA3 might interfere with apoptosis and proliferation of trophoblasts in PE. We used Western blot, immunofluorescence staining, and cell viability assays to evaluate how PDIA3 affects the apoptosis and proliferation of HTR-8 cells. Furthermore, we applied mRNA-sequencing assay and Western blot to investigate the gene expression and signaling pathway influenced by PDIA3. This represents the first study to explore the function of PDIA3 in the development of PE.

\section{Materials and methods}

\section{Patient samples}

Between February 2015 and April 2019, 13 placental trophoblasts specimens were collected from PE pregnancies and 13 specimens from normotensive pregnancies without complications treated at the Department of Obstetrics and Gynecology of the International Peace Maternity \& Child Health Hospital of the China Welfare Institute, Shanghai Jiao Tong University School of Medicine, China. PE was diagnosed according to the guidelines from The American College of Obstetricians and Gynecologists: new-onset hypertension (systolic blood pressure $\geq 140 \mathrm{mmHg}$ or diastolic blood pressure $\geq 90 \mathrm{mmHg}$ on two occasions at least $4 \mathrm{~h}$ ) after gestational week 20, with proteinuria, or with a new onset of the following: thrombocytopenia, renal insufficiency, impaired liver function, pulmonary edema, cerebral or visual disturbances (Gynecologists TAcooa 2019). Pregnant woman with chronic hypertension, renal disease, or other complications were not recruited. Comparing the clinical data from 13 preeclampsia pregnancies and 13 normotensive pregnancies, we found the blood pressure and 24-h urine protein in PE group were significantly higher than in control group, and gestational day at delivery was relatively lower in PE. The infants whose mothers with PE had lower birth weight and there were 3 samples complicated with fetal growth restriction in PE group. Patient demographic characteristics are summarized in Supplementary Table 1 (see section on supplementary materials given at the end of this article).

About $0.5 \mathrm{~cm}^{3}$ placental samples were collected from the central area of chorionic tissue located below one-third of the maternal side. The fresh tissues were washed immediately with PBS buffer, preserved in RNA later buffer at $-80^{\circ} \mathrm{C}$ for later RNA isolation. All samples for protein extraction were immediately frozen with liquid nitrogen and stored in $-80^{\circ} \mathrm{C}$ and were fixed overnight using $4 \%$ paraformaldehyde for $\mathrm{IHC}$ assay.

Written informed consent was obtained from all participants. Ethics approval for this study was granted by the Medical Ethics Committee of the International Peace Maternity \& Child Health Hospital of the China Welfare Institute.

\section{Quantitative real-time reverse transcription PCR (qRT-PCR)}

Total RNA was extracted from cultured cells or human placental villous tissues using the TRIzol reagent (Life Technologies), according to the manufacturer's instructions. After detecting the concentration and purity of the total RNA using a NanoDrop 2000c spectrophotometer (ThermoFisher Scientific), RNA was reverse-transcribed into cDNA using a PrimeScript ${ }^{\mathrm{TM}}$ II first Strand cDNA Synthesis Kit (Takara) with random or oligo-dT primers. Quantitative RT-PCR was performed using aSYBRGreen kit (Takara), and $0.4 \mu \mathrm{M}$ final concentration of primers was used, according to the manufacturer's protocol. The primers used were as follows: PDIA3 5-TGGATGCCACAGCCAATG-3 (forward) and 5- GGGTCCTAGTCCTCCCCAAT-3 (reverse); MDM2 5- GAATCATCGGACTCAGGTACATC-3 (forward) and 5- TCTGTCTCACTAATTGCTCTCCT-3 (reverse); GAPDH 5-CACTGGGCTACACTGAGCAC-3 (forward) and 5-AGTGGTCGTTGAGGGCAAT-3 (reverse). The primers used to detect mRNA expression levels after knockdown of PDIA3 are listed in Supplementary Table 2. For analysis of expression in clinical specimens, we computed relative gene expression using the $2^{-\triangle \mathrm{Ct}}$ method, normalized against GAPDH. For in vitro experiments, we calculated relative expression using the $2^{-\triangle \Delta C t}$ method, standardized to GAPDH.

\section{Western immunoblotting}

Cells and placental tissues were lysed for protein extraction using Radio Immunoprecipitation Assay (RIPA) lysis buffer. A BCA protein assay kit (Pierce Biotechnology) was utilized to detect the protein concentration in each sample, according to the manufacturer's protocol. Proteins were separated using SDS-PAGE and transferred onto $0.22 \mathrm{~mm}$ PVDF membranes. 
After blocking for $1 \mathrm{~h}$ in $5 \%$ non-fat milk with TBST buffer at room temperature, the membranes were incubated overnight at $4^{\circ} \mathrm{C}$ with the following specific antibodies diluted in Antibody Dilution Buffer (Epizyme Biotech, Shanghai, China): anti-PDIA3 (Abcam, diluted 1:1000), anti-MDM2 (Abcam, 2 $\mu \mathrm{g} / \mathrm{mL}$ ), anti-p53 (Cell Signaling Technology, diluted 1:1000), anti-cleaved caspase 3 (Abcam, diluted 1:1000), anti-P21 (Cell Signaling Technology, diluted 1:1000) and anti-GAPDH (Cell Signaling Technology, diluted 1:10,000). Membranes were then washed three times with TBST buffer for $5 \mathrm{~min}$ and were then incubated with the appropriate secondary antibodies for $1 \mathrm{~h}$. The membranes were then visualized using a Pro-light HRP chemiluminescent kit (Millipore Corporation) and a chemiluminescence detection system (Amersham imager 600; GE Healthcare Life Sciences), according to the manufacturer's instructions. The intensity of all protein bands was quantified using Image J software (NIH).

\section{Cell culture}

The immortalized HTR8/SVneo cell line (HTR-8, first-trimester human extravillous trophoblasts) was kindly provided by $\mathrm{Dr}$ PK Lala (University of Western Ontario, Canada). HTR-8 cells were cultured in RPMI1640 medium supplemented with 10\% fetal bovine serum (Gibco) in standard culture conditions (incubated at $37^{\circ} \mathrm{C}$ with $5 \% \mathrm{CO}_{2}$ atmosphere).

\section{Gene knockdown and overexpression}

About $3 \times 10^{5}$ cells per well were seeded onto six-well plates overnight and the cells were allowed to reach approximately $60-70 \%$ confluence prior to transient transfection. The specific siRNA targeting the sequence of the human PDIA3 and MDM2 mRNAs were siPDIA3-1 (5-GAGACUUGCACCUGAGUAUTT-3), SiPDIA3-2 (5-CCAGCAACUUGAGGGAUAATT-3), and SiMDM2 (5-AAUGCCUCAAUUCACAUAGAUUU-3), and siRNA oligonucleotides were designed and synthesized by GenePharma (Shanghai, China). siRNA oligonucleotides were transfected into HTR-8 cells at a final concentration of 100 $\mathrm{nmol} / \mathrm{L}$ using the Oligofectamine reagent (Invitrogen, Life Technologies), according to the manufacturer's instructions. To overexpress the PDIA3 and MDM2, plasmids including the coding region of human $P D I A 3$ or MDM2 were purchased from Genechem (Shanghai, China). The overexpression plasmids and the control vector were purified using the PureYield Plasmid Miniprep System (Promega), and then transfected into HTR-8 using Lipofectamine 3000 (Invitrogen), according to the manufacturer's recommendations. Cells were incubated for $48 \mathrm{~h}$ after transfection and were then harvested for Western blot and other analyses.

\section{Immunohistochemistry}

Immunohistochemistry (IHC) was performed using an EXPOSE Mouse and Rabbit-specific HRP/DAB Detection IHC kit (Abcam). Briefly, the fixed chorionic tissue samples were gradient alcohol dehydrated, then embedded, sectioned, deparaffinized and rehydrated in turn, and then antigen retrieval was performed by heating in a microwave oven in EDTA buffer, according to the manufacturer's recommendations. The slides were then incubated in 3\% hydrogen peroxide and blocked with $5 \%$ BSA. After incubation with primary antibodies diluted in PBS (anti-PDIA3 antibody 1:100, anti-MDM2 antibody 1:100, and anti-p21 antibody $1: 50$ ) overnight at $4{ }^{\circ} \mathrm{C}$, the slides were washed three times with PBS buffer and then incubated with the corresponding secondary antibodies. The tissues were stained using the DAB chromogen and counterstained with hematoxylin, and sections were then mounted with coverslips following dehydration. The images were captured under a Leica DMi8 microscope (Leica Microsystems) at $100 \times$ and $200 \times$ magnification. A semi-quantitative method was employed to calculate the protein expression, as previously described (Leandro Luongo de Matos et al. 2010, Mo et al. 2019).

\section{Immunofluorescence staining}

The siPDIA3-HTR8 cells were collected and re-cultured in 24-well plates overnight. Cells were washed twice with PBS and fixed with $4 \%$ paraformaldehyde for $20 \mathrm{~min}$, permeabilized with $0.5 \%$ Triton X-100 for $15 \mathrm{~min}$, and blocked with $5 \%$ BSA for $30 \mathrm{~min}$ at room temperature. Cells were incubated in primary antibody anti-Ki67 (Abcam, diluted 1:300 in PBS) at $4{ }^{\circ} \mathrm{C}$ overnight. Subsequently, cells were washed with PBS and incubated with Alexa Fluor 594-conjugated goat antiRabbit IgG (Life Technologies) antibodies for $1 \mathrm{~h}$ at room temperature in the dark. Finally, cells were counterstained with Fluoroshield mounting medium containing 4,6-diamidino2-phenylindole (DAPI; Abcam). Fluorescence microscopic images were obtained using a Leica DMi8 microscope (Leica Microsystems). The staining intensity was relatively quantified using ImageJ software (NIH).

\section{Flow cytometry analysis}

Knockdown or overexpressioned PDIA3-treated HTR-8 cells were harvested and stained with a fluorescein isothiocyanate (FITC)-Annexin-V Apoptosis Detection Kit with propidium iodide (Biolegend, San Diego, CA, USA) for $15 \mathrm{~min}$ in the dark, according to the manufacturer's protocol. Analysis of apoptosis was performed using flow cytometry (FACScan; BD Biosciences), and the relative ratios of apoptotic cells were counted for comparison using Flow Jo X software.

\section{Cell Counting Kit-8 assay}

After transient transfection with siRNAs or overexpressing plasmids for 48 h, HTR-8 cells were plated into 96-well plates at a density of $1.5 \times 10^{3}$ cells per well. Cell proliferation was assessed at 0, 24, 48, and $72 \mathrm{~h}$ utilizing the CCK8 assay (CCK8 assay, Dojindo, Kumamoto, Japan), according to the manufacturer's recommendations. Finally, the absorbance (optical density values) was detected at $450 \mathrm{~nm}$ using a Synergy H1 microplate reader (BioTek, Winooski, VT, USA). 


\section{EdU assay}

Cells were pre-incubated with $25 \mu \mathrm{M}$ 5-ethynyl-2'deoxyuridine (EdU) (Cell-Light EdU Apollo488 In Vitro Kit, RIBOBIO, Guangzhou, China) for $2 \mathrm{~h}$ in incubator. Then cells were fixed with $4 \%$ paraformaldehyde, permeabilized with $0.5 \%$ Triton X-100, and stained with the fluorescent dyes (Cell-Light EdU Apollo488 In Vitro Kit, RIBOBIO, Guangzhou, China), according to the manufacturer's instructions. Sections were visualized using a Leica DMi8 microscope (Leica Microsystems). The staining intensity was relatively quantified using ImageJ.

\section{mRNA sequencing (mRNA-seq)}

To identify the downstream mRNAs in siPDIA3-HTR8 cells compared with the control siRNA-treated group, total RNAs from the two treated groups were isolated using TRIzol Reagent (Invitrogen Life Technologies). Three micrograms of RNA from each sample was used as input material for the RNA sample preparations. Sequencing libraries were generated using the TruSeq RNA Sample Preparation Kit (Illumina). The sequencing and data analyses were performed by Personal Biotechnology (Shanghai, China). The threshold value for significance was fold change $>1.5$ with a $P$ value $<0.05$. Details about the specific methods are provided in Supplementary RNAsequencing method.

\section{Statistical analysis}

Statistical analysis was performed using GraphPad Prism, version 6.0 (GraphPad). For parametric data, Student $t$-tests were used to compare two groups and one-way ANOVA tests were used to analyze multiple groups. For nonparametric data, Mann-Whitney tests for 2 groups, Kruskal-Wallis tests for multiple groups, respectively. All in vitro experiments were repeated three times and the data were presented as median with range. All $P$ values are two-sided, and $P$ value $<0.05$ was considered statistically significant.

\section{Results}

\section{PDIA3 is decreased in trophoblasts from patients with preeclampsia}

We investigated the expression level of PDIA3 in villous tissues from PE vs normotensive pregnancies (NP). The results of quantitative RT-PCR and Western blot analysis showed that PDIA3 protein and mRNA expression were significantly downregulated in the placental trophoblasts of patients with PE compared to NP (Fig. 1A, B and C). Furthermore, immunohistochemical (IHC) analyses were conducted in 26 villous tissue samples (13 PE samples and $13 \mathrm{NP}$ samples) to investigate the localization and expression levels of PDIA3. In IHC analyses, the staining intensity of PDIA3 was significantly weaker in villous tissues from the PE group than the NP group, and PDIA3-positive cells were detected mainly in the syncytiotrophoblasts (Fig. 1D and E). Collectively, these results indicate that $\mathrm{PDIA} 3$ expression is decreased in villous trophoblasts from PE pregnancies.

\section{Knockdown of PDIA3 induces apoptosis and impairs proliferation in HTR-8 cells}

We tested PDIA3 protein expression level in available JAR, Bewo and HTR8 cell line (Fig. 2A). Bewo has the highest expression level of PDIA3, but Bewo cell is mainly used in trophoblast secretion and fusion experiments, and considering PE is trophoblasts-origin disease and HTR8 cell is widely used in trophoblast proliferation and invasion experiments, we used HTR8 cells to perform in vitro experiments. To evaluate the
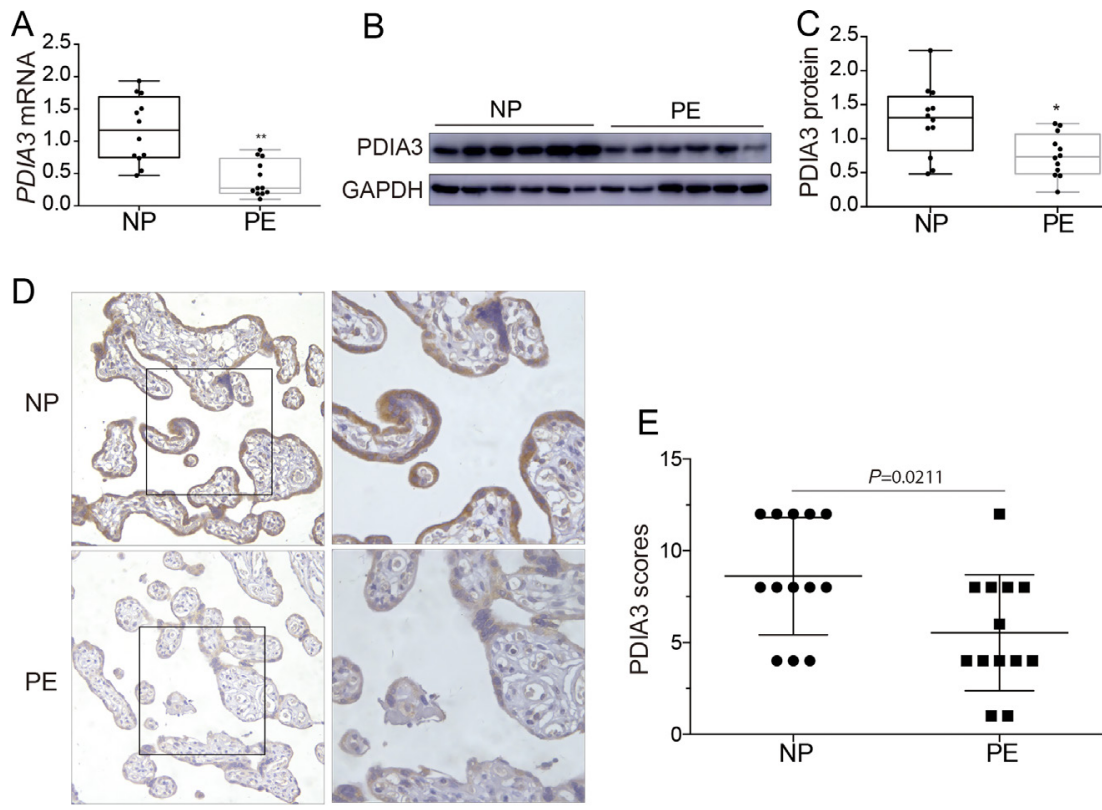

Figure 1 Decreased PDIA3 in preeclampsia (PE) vs normotensive pregnancies. (A) The relative mRNA levels of PDIA3 in placental trophoblasts of PE pregnancies compared to normotensive pregnancies (NP). GAPDH served as a loading control. (B) Representative images of western blot showed PDIA3 protein levels of placental trophoblasts, $n=6$. (C) Statistical comparison of PDIA3 protein level in the PE and NP groups, $n=12$. (D and $\mathrm{E}$ ) Representative images of IHC staining for PDIA3 and statistical comparison of scores in the PE and NP groups. Magnification 200x (left), $n=13$. For data in A, C and E, Student t-tests were used, and the data were presented as mean \pm S.D. ${ }^{*} P<0.05,{ }^{* *} P<0.01$. 
A

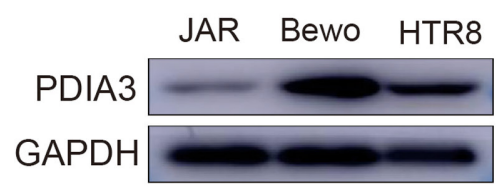

D

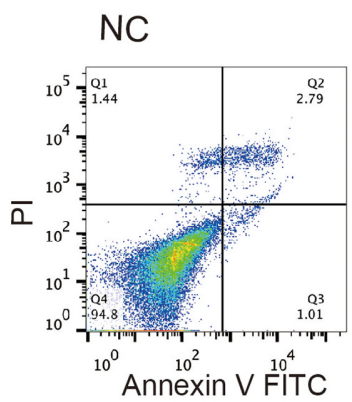

$\mathrm{F}$

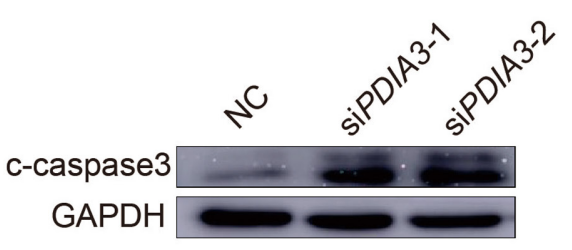

G

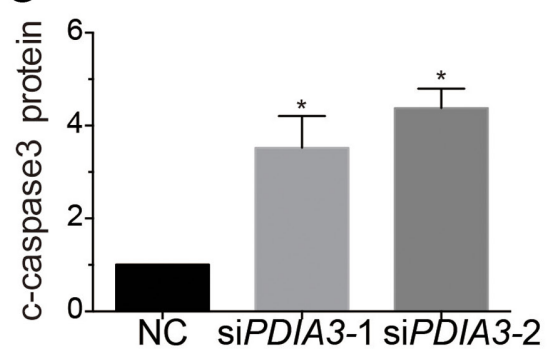

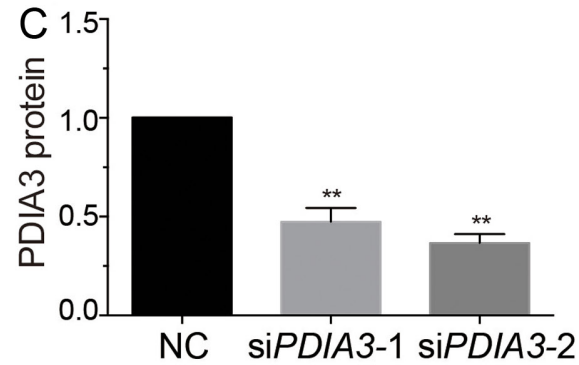

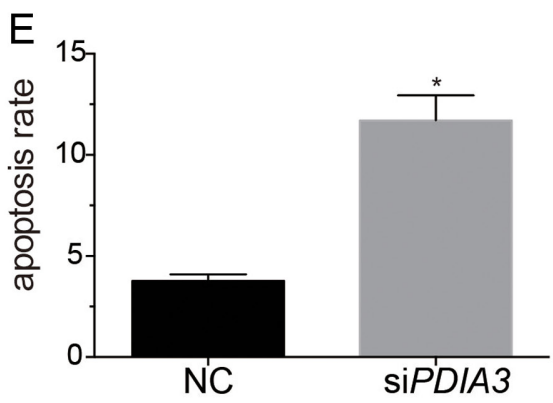

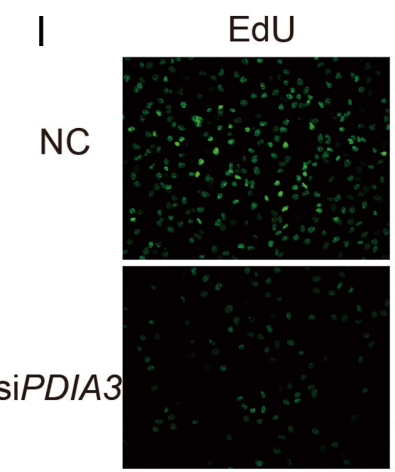
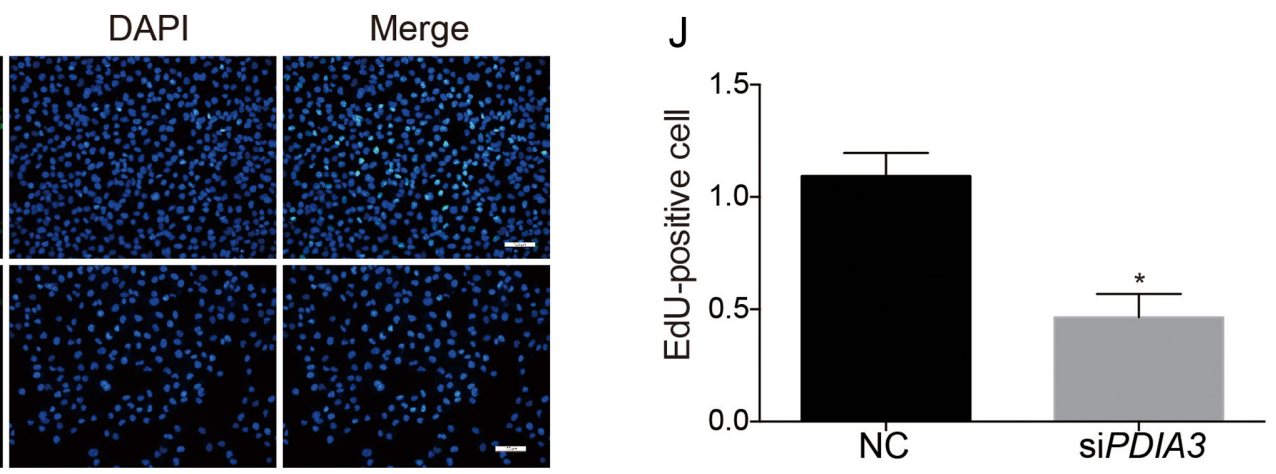

Figure 2 Knockdown of PDIA3 promotes apoptosis and impairs proliferation in HTR-8 cells. (A) PDIA3 protein level in JAR, Bewo and HTR-8 cells. (B and C) Verifying the knockdown of PDIA3 in HTR-8 cells treated with siPDIA3 oligonucleotides. (D and E) The relative apoptosis rate of HTR-8 cells was determined by flow cytometry analysis after cells were treated with negative control (NC) or siPDIA3 (siPDIA3-2). (F and G) The effects of PDIA3 knockdown on the protein level of cleaved caspase 3 was analyzed by Western blot. (H) Cell Counting Kit-8 assay showed that decreased PDIA3 impairs HTR-8 proliferation. (I and J) EdU assay showing incorporation of EdU-label into HTR-8 cells (green). Original magnification $200 \times,{ }^{*} P<0.05 ;{ }^{* *} P<0.01 ;{ }^{* * *} P<0.001$ vs NC, each in vitro test was performed three times, for data in graph $\mathrm{C}, \mathrm{E}, \mathrm{G}, \mathrm{H}$ and $\mathrm{J}$, Mann-Whitney tests were used, and the data were presented as median with range. NC: transfection of negative control (NC) only in HTR-8; SIPDIA3: transfections of siPDIA3-2 in HTR8; siPDIA3-1/siPDIA3-2: transfections of siPDAI3-1 or siPDIA3-2 alone in HTR-8. 

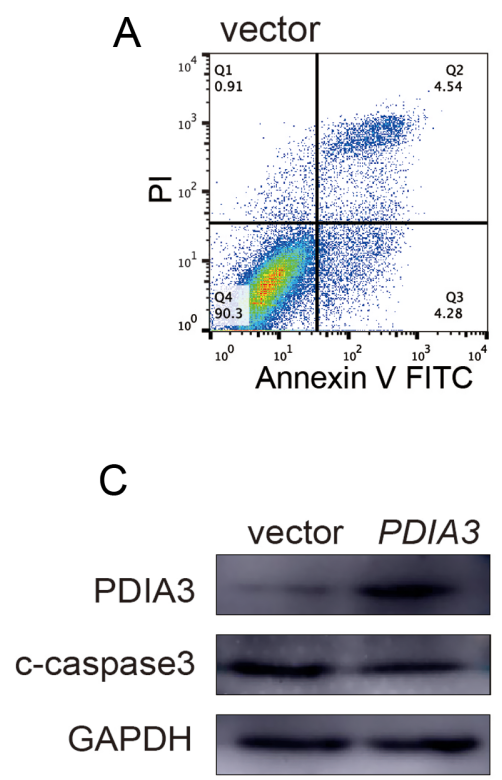

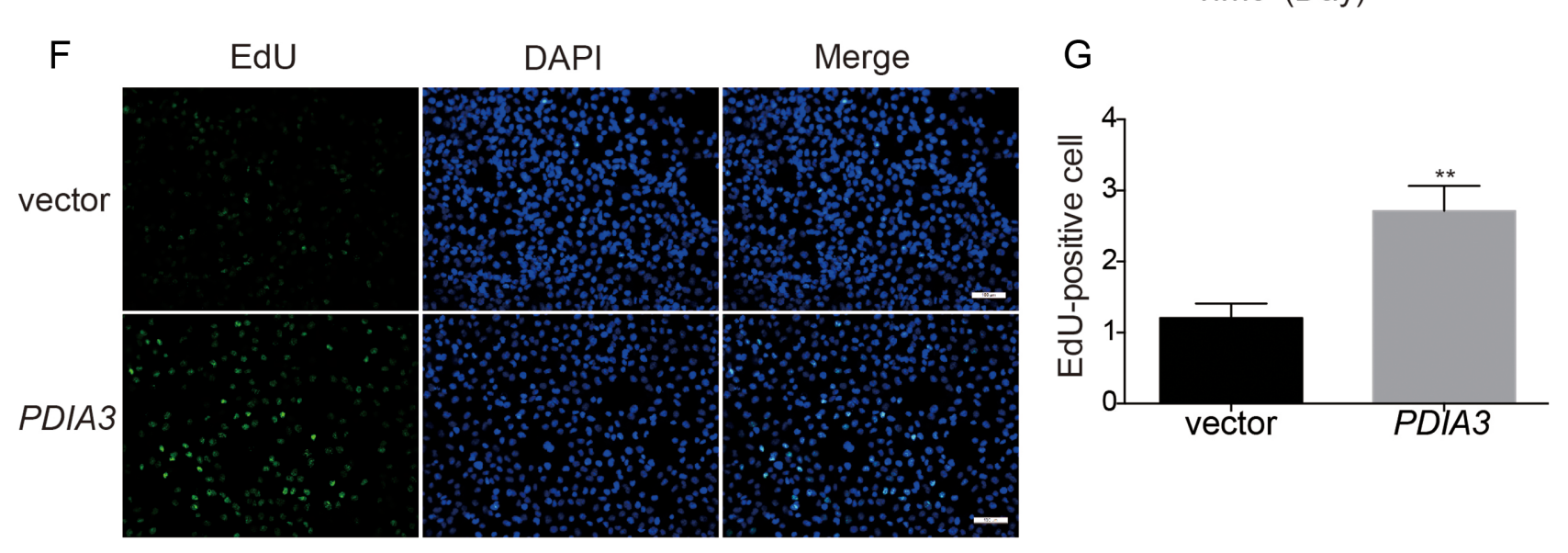

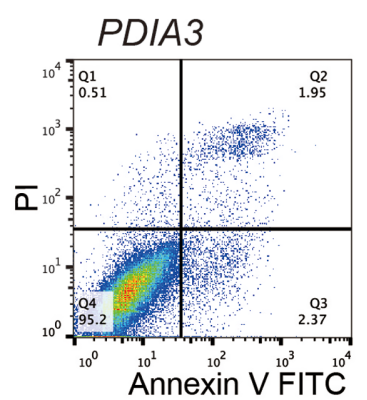

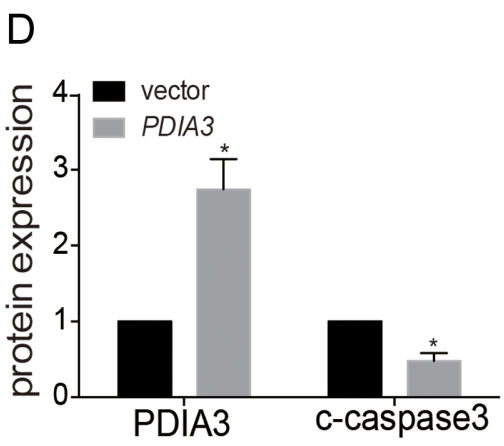

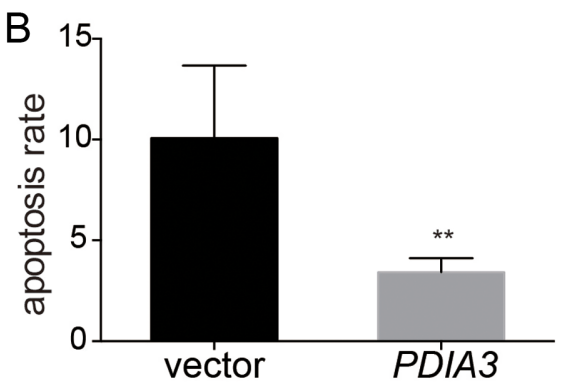

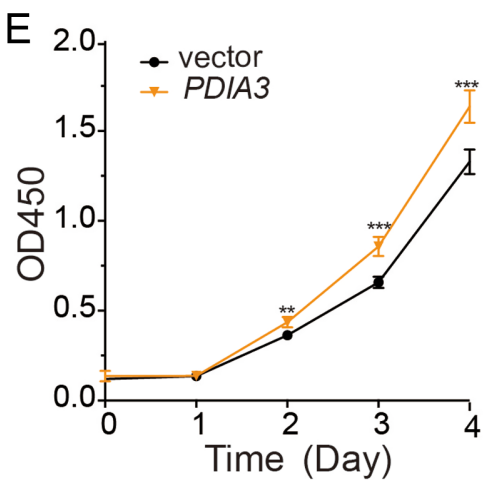

Figure 3 Overexpression of PDIA3 reduces apoptosis and promotes proliferation in HTR-8 cells. (A and B) The relative apoptosis rate of HTR-8 cells was determined by flow cytometry analysis after transfection with PDIA3 or control vector. (C and D) Western blot assay showed the effects of overexpression of PDIA3 on protein expression of cleaved caspase 3. (E) Cell Counting Kit-8 assay showed that increased PDIA3 promotes HTR-8 proliferation. ( $F$ and G) EdU assay detected the proliferation level of HTR-8 cells when transfected with PDIA3 overexpression plasmid. Original magnification $200 \times .{ }^{*} P<0.05 ;{ }^{*} P<0.01 ;{ }^{* * *} P<0.001$ vs vector, each in vitro test was performed three times, for data in graph $\mathrm{B}, \mathrm{D}$, $E$ and G, Mann-Whitney tests were used, and the data were presented as median with range. vector: cells transfected with vector control. PDIA3: transfection of plasmid of PDIA3.

role of PDIA3 in the proliferation and apoptosis of trophoblasts, we transfected negative control (NC) siRNA and siRNA against PDIA3 into human trophoblastderived HTR-8 cells. Western blot analysis showed that PDIA3 protein expression was markedly decreased after transfection with siPDIA3-1 and siPDIA3-2 (Fig. 2B and C). We selected siPDIA3-2 for subsequent knockdown experiments. Flow cytometric analysis of cell apoptosis by annexin-V/propidium iodide (PI) staining, showed that PDIA3 knockdown increased HTR-8 cell apoptosis
(Fig. 2D and E). Western blot showed that expression of the apoptotic marker cleaved-caspase 3 protein was significantly increased following knockdown of PDIA3 (Fig. 2F and G). The cell counting kit-8 (CCK8) assay showed that PDIA3 knockdown impaired HTR-8 cell proliferation (Fig. $2 \mathrm{H}$ ). 5-ethynyl-2-deoxyuridine (EdU; $\mathrm{RIBOBIO)}$ was used to examine cell proliferation. Knockdown of PDIA3 reduced the proportion of EdUlabeled cells (Fig. $2 \mathrm{I}$ and J). Furthermore, IF staining of proliferative marker Ki67 in siPDIA3-HTR8 cells showed 
that Ki67 was more weakly expressed in siPDIA3-HTR8 cells (Supplementary Fig. 1). These results indicate that knockdown of PDIA3 promoted cell apoptosis and inhibited proliferation of HTR-8 cells.

\section{Overexpression of PDIA3 reduces apoptosis and induces proliferation of HTR-8 cells}

Wetransfected PDIA3 overexpression plasmid into HTR-8 cells to investigate the effects of PDIA3 overexpression on trophoblast apoptosis and proliferation. Flow cytometry showed that overexpression of PDIA3 can reduce HTR-8 cell apoptosis (Fig. 3A and B). Western blot revealed that PDIA3 protein expression levels were significantly upregulated after overexpressing PDIA3, and expression of the apoptotic marker cleaved-caspase 3 was decreased, compared with the vector-transfected cells (Fig. 3C and D). The CCK8 assay showed that increased PDIA3 increased HTR-8 proliferation (Fig. $3 \mathrm{E})$. EdU staining in HTR-8 cells revealed an increase in EdU-label cells following overexpression of PDIA3 (Fig. $3 F$ and $G$ ). These results verify that PDIA3 is involved in
HTR-8 cells apoptosis and proliferation and suggest that PDIA3 positively regulates proliferation in trophoblasts.

\section{PDIA3 regulates the MDM2/p53 signaling pathway in HTR-8 cells}

To further explore the mechanism of action by which PDIA3 regulates trophoblast apoptosis and proliferation, we used next-generation mRNA sequencing (RNA-seq) technology to detect transcriptomic changes of three paired HTR-8 cell samples transfected with siPDIA3 or control siRNA. A total of 115 differentially expressed genes were identified after knockdown of PDIA3 (Supplementary xlx3). Of these 115 genes, 68 genes were upregulated and 47 were downregulated (Fig. 4A and B). We performed KEGG enrichment analysis of these differential expression genes (Supplementary Fig. 2 ) and found that the differentially expressed genes were enriched in pathways involved in regulation of actin cytoskeleton, apoptosis, and p53 signaling pathway, and p53 signal pathway-responsible cellular processes (Fig. 4C). We found that MDM2 mRNA was upregulated
A

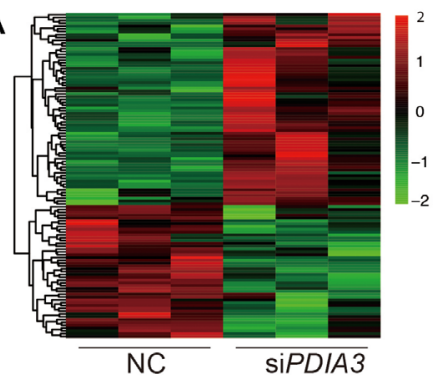

C
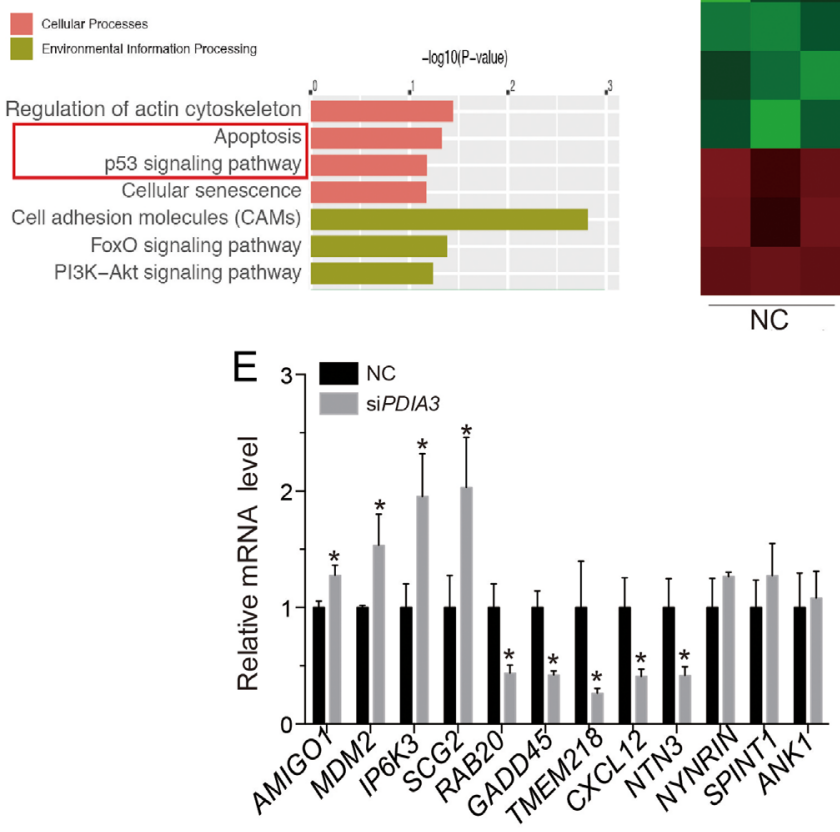

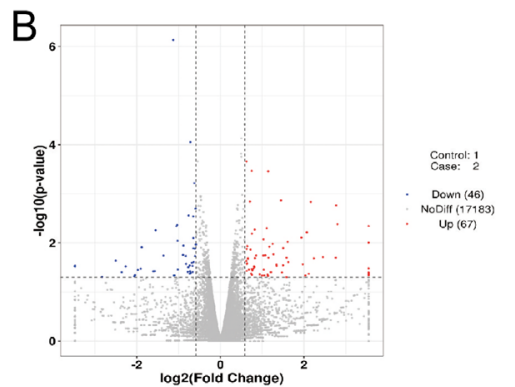

D

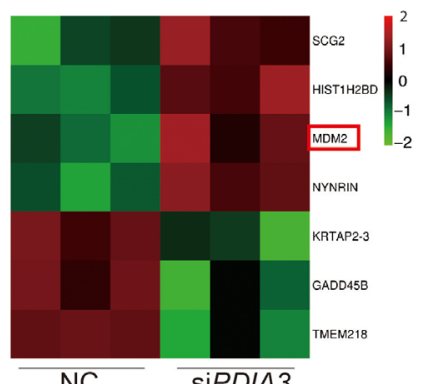

NC $\quad \overline{\text { siPDIA3 }}$

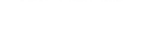

Figure 4 RNA sequencing indicated that decreased PDIA3 alters MDM2 and $p 53$ related genes. (A and $B$ ) Heatmap $(A)$ and volcano plot (B) showing differentially expressed genes after knockdown of PDIA3. (C) KEGG pathway enrichment analysis including dysregulated genes regarding cellular processes and environmental information processing. (D) Heatmap showing mRNA levels of the seven selected dysregulated genes. (E) RT-PCR assay verified the mRNA expression levels of 12 selected differentially expressed genes, $n=3$, MannWhitney tests were used, and the data were presented as median with range. ${ }^{*} P<0.05$. 

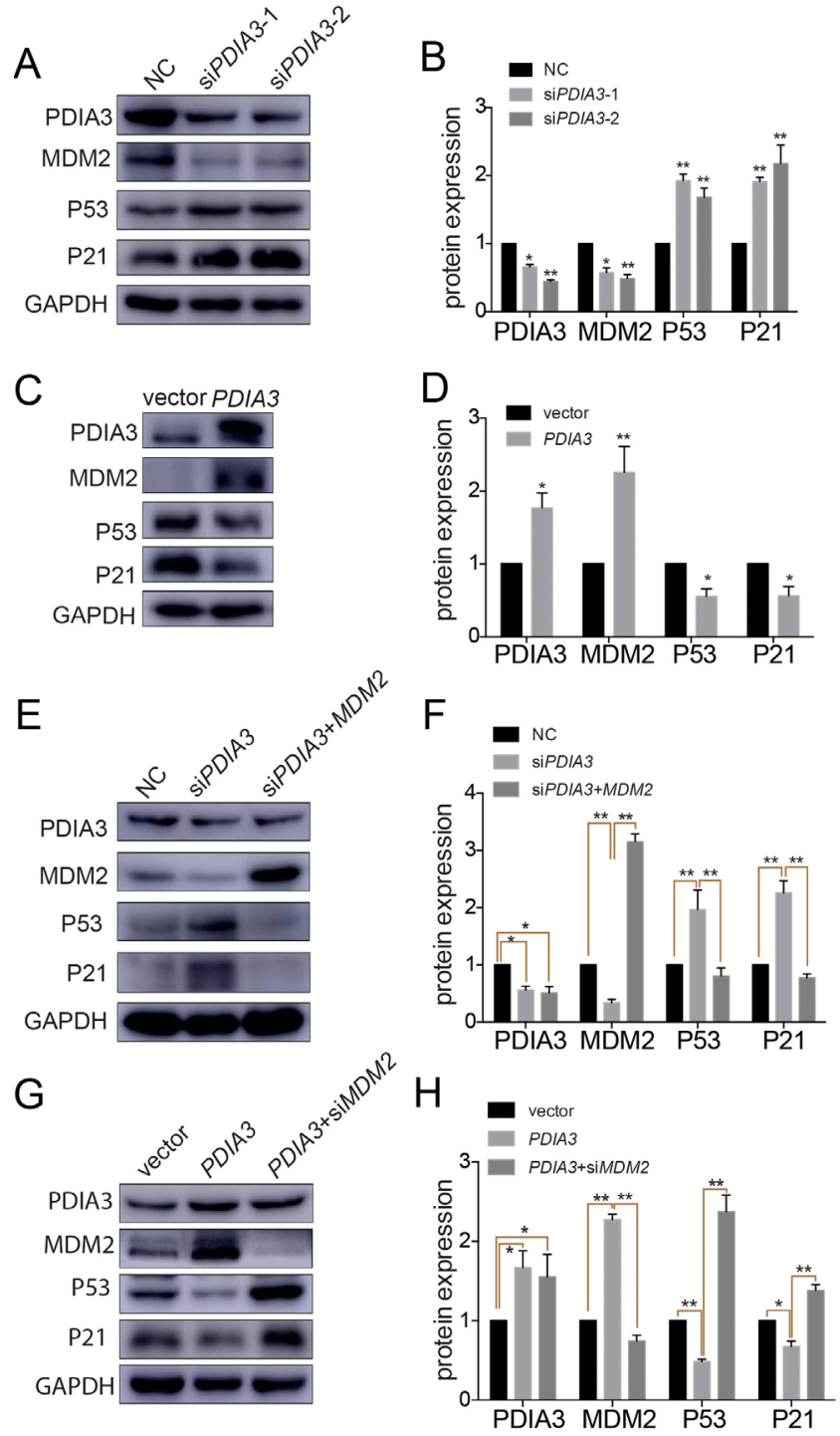

Figure 5 PDIA3 regulates MDM2/p53/p21 pathway in HTR-8 cells. (A and B) Western blot showing the protein expression levels of MDM2, p53, and p21 after knockdown of PDIA3 in HTR-8 cells. (C and D) The expression of MDM2, p53, and p21 after overexpression of PDIA3 in HTR-8 cells. (E and F) The expression of MDM2, p53, and p21 were analyzed by Western blot in MDM2-overexpressing HTR-8 cells treated with siPDIA3. (G and $\mathrm{H}$ ) The expression of MDM2, p53, and p21 after suppression of MDM2 in PDIA3-overexpressing HTR8 cells. Each test was performed three times, Mann-Whitney tests were used in graph $\mathrm{B}, \mathrm{D}, \mathrm{F}$ and $\mathrm{H}$, and the data were presented as median with range. ${ }^{*} P<0.05 ;{ }^{* *} P<0.01$.

1.68-fold after PDIA3 knockdown (Fig. 4D). We selected 12 out of 115 differentially expressed genes to verify the mRNA levels in HTR-8 cells transfected with siPDIA3 or NC (Fig. 4E).

Considering previous assays showed that apoptosis and proliferation of HTR-8 cells were affected by transfection with $P D I A 3$ siRNA, we selected the MDM2 gene to confirm whether MDM2 was specifically regulated by PDIA3. We found that MDM2 protein levels were decreased after knockdown of PDIA3, as indicated by Western blot, and the downstream p53 and p21 changed correspondingly (Fig. 5A and B). Overexpression of PDIA3 induced MDM2 protein expression and regulated p53 and p21 correspondingly (Fig. 5C and D). In addition, the upregulation of the apoptosis-related genes p53 and p21 caused by PDIA3 suppression was reversed by MDM2 overexpression (Fig. $5 \mathrm{E}$ and $\mathrm{F}$ ). In contrast, knockdown of MDM2 rescued the expression of p53 and p21 after PDIA3 overexpression (Fig. 5G and $\mathrm{H}$ ).

We performed flow cytometry analysis and EdU assay to verify the functions of apoptosis and proliferation in HTR-8 cells. Flow cytometry analysis showed that the MDM2 overexpression rescued the effect of apoptosis after PDIA3 suppression, and knockdown of MDM2 reversed the apoptosis rate in trophoblasts after PDIA3 overexpression (Fig. 6A, B, C and D). Correspondingly, $\mathrm{EdU}$ assay showed that upregulation of MDM2 rescued the proliferation function caused by knockdown of PDIA3 in HTR8, and MDM2 suppression reversed the proliferation after PDIA3 overexpression (Fig. 6E, F, $\mathrm{G}$ and $\mathrm{H}$ ). From these data, we conclude that PDIA3 regulates apoptosis and proliferation of HTR-8 cells through the MDM2-p53 pathway.

\section{Trophoblasts from patients with preeclampsia exhibit decreased MDM2 protein expression and increased p21 expression}

We evaluated expression of MDM2, p53 and the downstream related protein, p21, in villous tissue samples from PE and NP pregnancies. RT-PCR assessment showed that both MDM2 and p53 mRNA levels were increased in placental villous tissues from PE pregnancies compared to NP pregnancies (Fig. 7A). Western blot showed that $\mathrm{p} 53$ protein was increased, but MDM2 protein expression was significantly decreased in PE pregnancies (Fig. 7B and C). IHC assessment showed that the expression of MDM2 was located in placental syncytiotrophoblast, and showed considerably weaker expression in PE tissues compared to NP (Fig. 7D and E). Moreover, IHC and western blot also showed that p21 was significantly differentially expressed in PE trophoblasts (Fig. 7F, G and H). Our results reveal that p21 was related to enhanced apoptosis of trophoblasts in PE. We verified that the MDM2 protein was downregulated and trophoblast apoptosis was enhanced in $\mathrm{PE}$ compared to NP.

\section{Discussion}

Many efforts have been made to increase our understanding of the pathogenesis of preeclampsia, and there is a consensus that dysfunctional trophoblasts, which affect invasion and remodeling of decidual spiral 


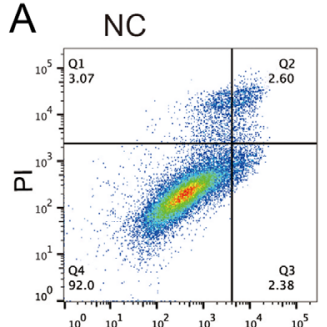

Annexin V-FITC

C

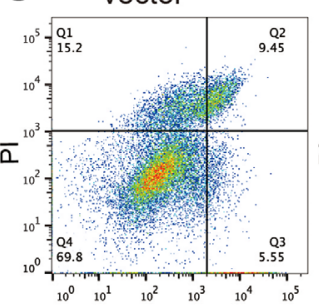

Annexin V-FITC

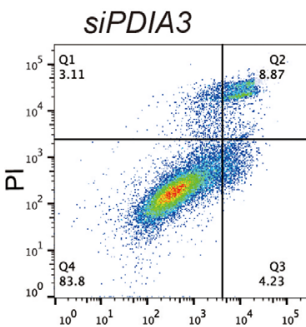

Annexin V-FITC

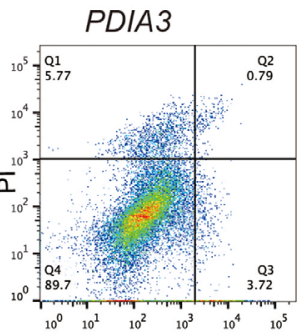

Annexin V-FITC

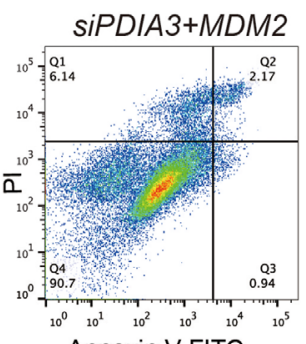

Annexin V-FITC

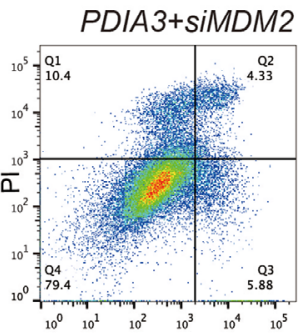

Annexin V-FITC
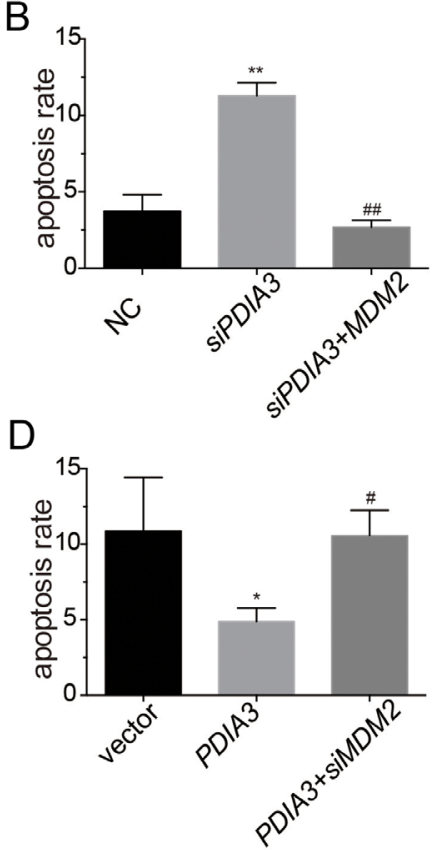

$\mathrm{E}$

EdU

DAPI

Merge

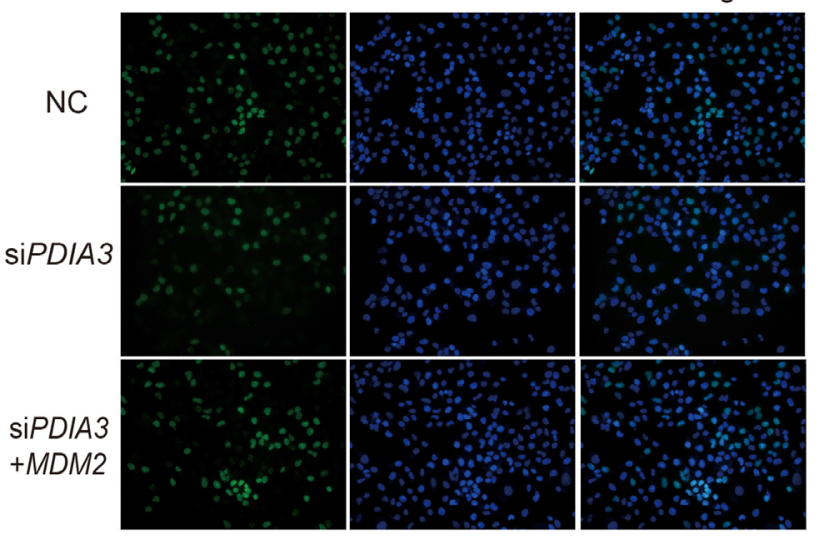

F

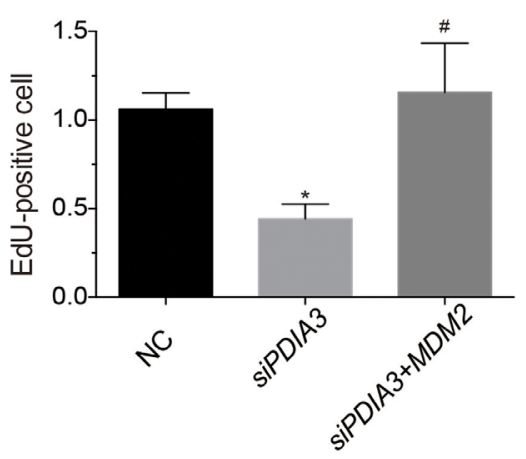

G

EdU

DAPI

Merge
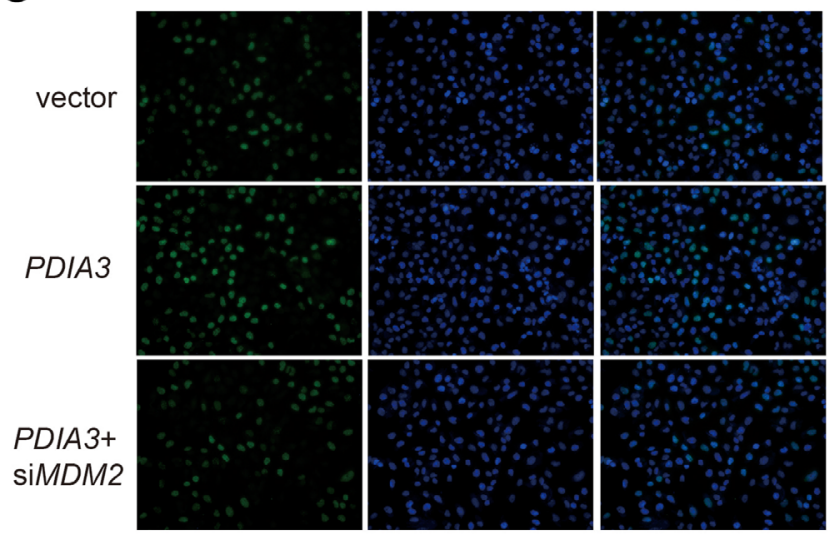

$\mathrm{H}$

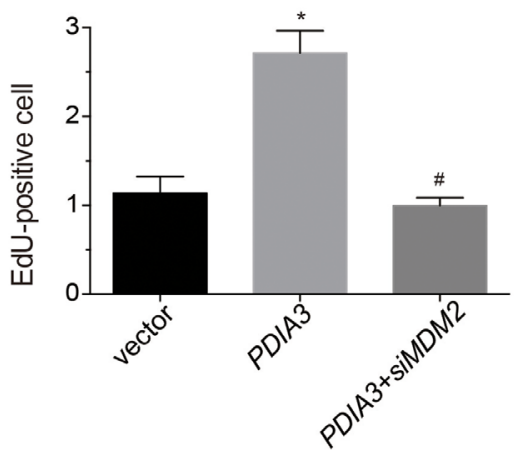

Figure 6 PDIA3 affects apoptosis and proliferation by regulating MDM2 in HTR-8 cells. (A, B, C and D) Flow cytometry analysis showing the relative apoptosis rate of HTR-8 cells after cells were transfected 48 h. (E, F, G and H) EdU assay showing incorporation of EdU-label into HTR-8 cells (green). Original magnification $200 \times, * P<0.05 ; * *<0.01$ vs control group, $\# P<0.05 ; \# \#<0.01$ vs siPDIA/PDIA3, each in vitro test was performed three times, Mann-Whitney tests were used in graph B, D, F and $\mathrm{H}$, and the data were presented as median with range. NC: transfection of negative control (NC, control group) in HTR-8; siPDIA3: transfections of siPDIA3-2, siPDIA3+MDM2 (co-transfected with SiPDIA3-2 and plasmid of MDM2), vector: cells transfected with vector control, PDIA3: transfection of plasmid of PDIA3, PDIA3+siMDM2 (co-transfected with plasmid of PDIA3 and siMDM2). 
A

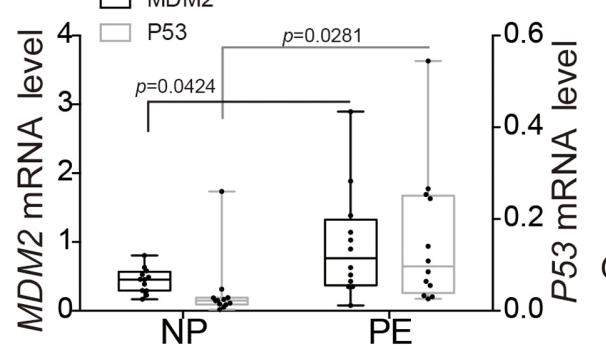

C

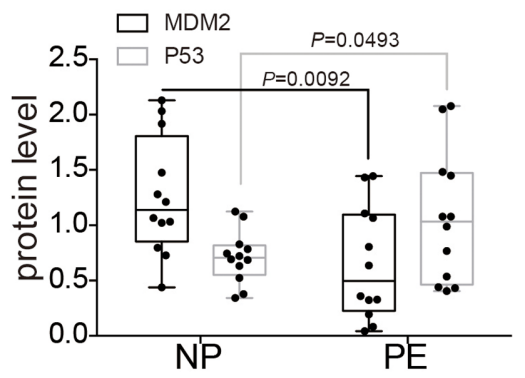

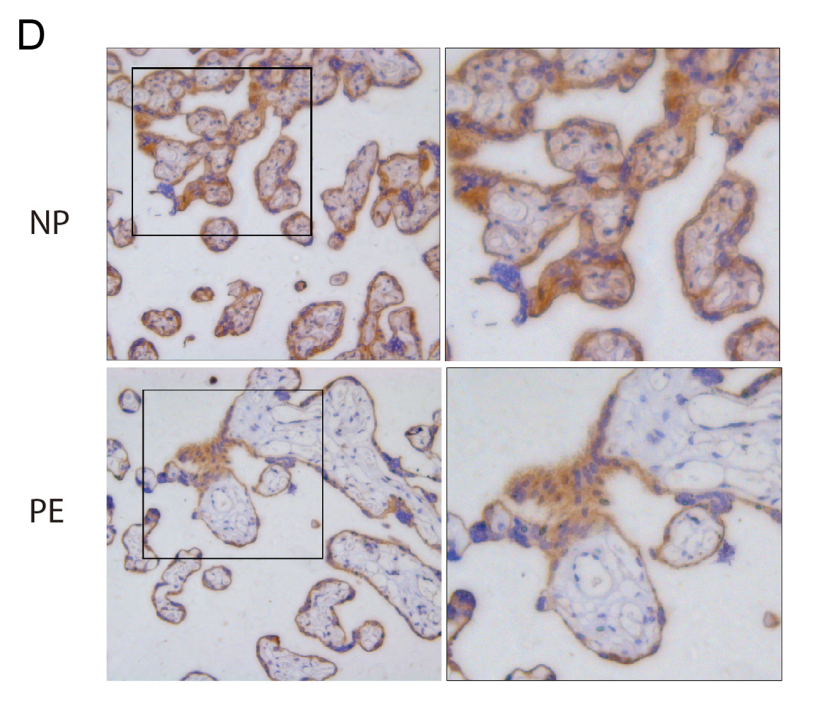

$E$

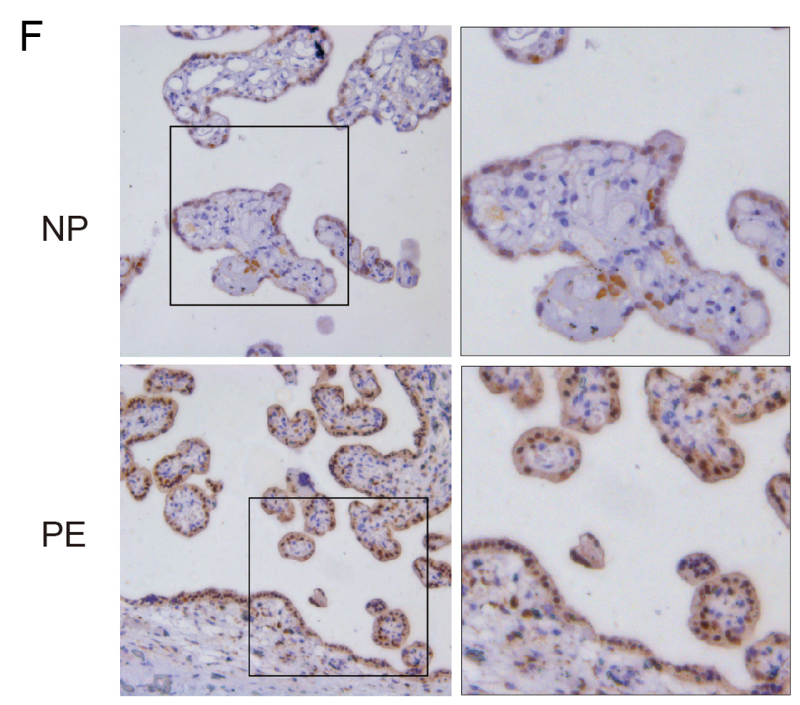

G
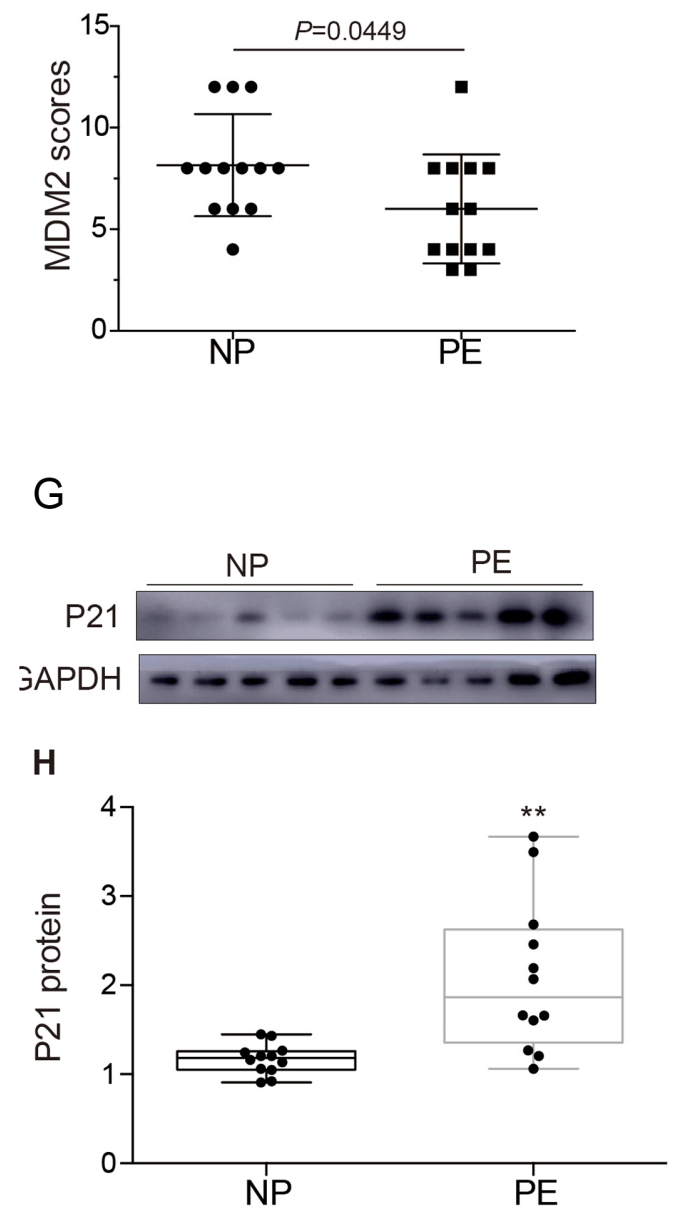

Figure 7 Abnormal expression of MDM2 and increased p21 in PE placental trophoblasts. (A, B and C) RT-PCR assay (A) detecting MDM2 and p53 relative mRNA levels and western blot analysis (B and C) of MDM2 and p53 protein levels in placental tissues from normotensive pregnancies and PE pregnancies, $n=12$ in A and C, $n=5$ in B. (D and E) Immunohistochemical staining was performed to detect the localization and expression of MDM2 in human placental trophoblasts, and statistical scores were analyzed, $n=13$ in $\mathrm{E}$. $(\mathrm{F}-\mathrm{H}) \mathrm{IHC}$ staining $(\mathrm{F})$ and Western blot analysis $(\mathrm{G}$ and $\mathrm{H})$ showed the localization and relative expression levels of p21 in placental trophoblasts, $n=5$ in $\mathrm{G}$ and $n=12$ in $\mathrm{H}$. For data in graph A, C, E and H, Student t-tests were used to compare two groups, and the data were presented as mean \pm S.D. Magnification 200x. $* P<0.05,{ }^{* *} P<0.05$. 
arteries, are important contributors to the development of PE. A quantitative assessment of placental apoptosis by electron microscopy confirmed a rise in placental apoptosis with pregnancy progression (Smith et al. 2000). Longtine et al. found significantly raised apoptosis in cytotrophoblasts from women with PE compared to term, normotensive pregnancies (Longtine et al. 2012). Increased apoptosis was also observed in syncytiotrophoblasts in term placentas complicated with PE (Naonori Ishihara et al. 2002). In PE, increased apoptosis of trophoblasts is a remarkable pathological characteristics. In this study, we found that PDIA3 was decreased in placental villous tissues from women with PE compared to normotensive pregnancies. Another major finding was that knockdown of PDIA3 promoted apoptosis and inhibited proliferation in HTR-8 cell line, a trophoblast cell-line model.

Previously, $\mathrm{Xu}$ et al. reported that knockdown of PDIA3 can protect endothelial cells from hyperoxia- or tunicamycin-induced apoptosis by inhibiting caspase-3 activation (Xu et al. 2009). Pressinotti $\mathrm{N}$ et al. reported that PDIA3 has a pro-apoptotic role in prostate cancer cells(Pressinotti et al. 2009). On the contrary, our study found that cleaved caspase- 3 expression was more than 3-fold-change upregulated following knockdown of PDIA3, which is consistent with a previous report that suppressed expression of PDIA3 resulted in increased cleaved caspase-3 protein expression and an increase in the sub-G1 population of rat insulinoma INS-1 cells (Yamamoto et al. 2014). Furthermore, using flow cytometry, CCK8 assay, and IF staining, we verified that knockdown of PDIA3 resulted in growth inhibition and apoptosis of HTR-8 cells. The contradictory result may suggest that PDIA3-mediated activation of apoptotic and proliferative pathways varies among different cell lines and disease models. HTR-8 cells are often used as a model to study EVTs, but there may be no suitable cell lines derived from cytotrophoblasts, since EVTs come from a subpopulation of cytotrophoblasts and also express PDIA3. Determining the effects of PDIA3 on different cells and disease models will elucidate the role of PDIA3 in different contexts.

The MDM2/p53 signaling pathway is an important regulatory constituent of apoptosis. The absence of MDM2 leads to the activation of the p53-dependent apoptotic pathway in mouse embryonic fibroblasts (Sohela de Rozieres et al. 2000). Here, we used RNA sequencing to identify genes that were differentially expressed after knockdown of PDIA3 in HTR-8 cells. Among those genes, we found that expression of the MDM2 gene was upregulated 1.68-fold-changed and was associated with apoptosis and proliferation. The inconsistency between protein and mRNA levels of MDM2, may be due to the p53-MDM2 self-feedback regulation, wherein mRNA transcription of MDM2 is upregulated by increased p53 protein (D. A. Freedman et al. 1999, Lahav et al. 2004). We predict that PDIA3 may regulate translation of MDM2 or MDM2 protein degradation, revealing a new biological mechanism involved in the pathology of PE. Collectively, our results are the first to demonstrate that that PDIA3 positively regulated MDM2 protein levels in HTR-8 cells, which is related to the pro-apoptosis properties of p53.

For clinical samples, we also detected increased MDM2 mRNA levels and decreased MDM2 protein expression in PE villous tissues compared to tissues from normal pregnancies. Additionally, we found increased p53 and p21 protein levels in PE placental tissues, which corroborates the previous reports indicating increased apoptosis is involved in PE (Afroze et al. 2016). Our results identified that the PDIA3-MDM2/p53 pathway was involved in the pathology of PE and suggest that increased apoptosis in villous tissues is an important potential diagnostic features in PE. We need to note that the collected tissues mainly contained cytotrophoblasts, syncytiotrophoblasts, and villous interstitium. Besides, the limited sample size and inevitable individual differences made our results relatively credible. Combined with the HTR-8 cell in vitro experiments, our clinical results become more persuasive. PDIA3 is detected at different cellular localizations, and has multiple functions (Hettinghouse et al. 2018). Future studies that explore function of PDIA3 in different cellular localizations within trophoblasts, a larger epidemiological survey and animal experiments that investigate the function of PDIA3 using in vivo models. will be needed to fully elucidate the roles of PDIA3 in PE.

In conclusion, we show that PDIA3 is significantly decreased in placental villous tissues during PE. Our results lend credence to the idea that decreased PDIA3 can promote apoptosis and impair proliferation in trophoblasts, and first demonstrate that PDIA3 regulates trophoblast apoptosis and proliferation through the MDM2-p53 signal pathway. Furthermore, we first report the discovery of a novel PDIA3/MDM2/p53 regulatory pathway that is involved in pathological process of $\mathrm{PE}$, and we suggest that PDIA3 could be a potential diagnostic and therapeutic target in PE.

\section{Supplementary materials}

This is linked to the online version of the paper at https://doi. org/10.1530/REP-200-0156.

\section{Declaration of interest}

The authors declare that there is no conflict of interest that could be perceived as prejudicing the impartiality of the research reported. 


\section{Funding}

This work was supported by the National Key Research and Development Program of China (2018YFC1002800), the Special Funds for Local Science and Technology Development Guided by the Central Committee (2018ZYYD014), the Clinical Research Fund of the International Peace Maternity and Child Health Hospital, Shanghai Jiao Tong University School of Medicine (GFY5816), and the Leading Fund of Renmin Hospital of Wuhan University (RMYD2018M75). The authors would like to thank the Department of Biobank (International Peace Maternity and Child Health Hospital, Shanghai Jiao Tong University) for providing the clinical samples.

\section{Author contribution statement}

$\mathrm{H} Q \mathrm{M}$ and $\mathrm{F} J \mathrm{~T}$ conceived and designed the experiments. $\mathrm{H}$ Q M, X L M, Y C Z, C X Z and W H Z helped to perform the experiments. $\mathrm{MHQ}$ and F JT analyzed the data and wrote the manuscript, $Y Z$ and $Y L$ contributed reagents and materials and to revise the manuscript.

\section{References}

ACOG practice bulletin no.202 2019 Gestational hypertension and preeclampsia. Obstetrics and Gynecology 133 e1-e25. (https://doi. org/10.1097/AOG.0000000000003018)

Afroze SH, Kalagiri RR, Reyes M, Zimmerman JD, Beeram MR, Drever N, Zawieja DC, Kuehl TJ \& Uddin MN 2016 Apoptotic and stress signaling markers are augmented in preeclamptic placenta and umbilical cord. BBA Clinical 6 25-30. (https://doi.org/10.1016/j. bbacli.2016.05.003)

Aureli C, Gaucci E, Arcangeli V, Grillo C, Eufemi M \& Chichiarelli S 2013 ERp57/PDIA3 binds specific DNA fragments in a melanoma cell line. Gene 524 390-395. (https://doi.org/10.1016/j.gene.2013.04.004)

Chamberlain N, Korwin-Mihavics BR, Nakada EM, Bruno SR, Heppner DE, Chapman DG, Hoffman SM, van der Vliet A, Suratt BT, Dienz O et al. 2019 Lung epithelial protein disulfide isomerase A3 (PDIA3) plays an important role in influenza infection, inflammation, and airway mechanics. Redox Biology 22 101129. (https://doi.org/10.1016/j. redox.2019.101129)

de Rozieres S, Maya R, Oren M \& Lozano G 2000 The loss of mdm2 induces p53 mediated apoptosis. Oncogene 19 1691-1697. (https://doi. org/10.1038/sj.onc.1203468)

DiFederico E, Genbacev O \& Fisher SJ 1999 Preeclampsia is associated with widespread apoptosis of placental cytotrophoblasts within the uterine wall. American Journal of Pathology 155 293-301. (https://doi. org/10.1016/S0002-9440(10)65123-1)

Duley L 2009 The global impact of pre-eclampsia and eclampsia. Seminars in Perinatology 33 130-137. (https://doi.org/10.1053/j. semperi.2009.02.010)

Freedman DA, Wu L \& Levine AJ 1999 Functions of the MDM2 oncoprotein. Cellular and Molecular Life Sciences 55 96-107. (https:// doi.org/10.1007/s000180050273)

Grillo C, D'Ambrosio C, Scaloni A, Maceroni M, Merluzzi S, Turano C \& Altieri F 2006 Cooperative activity of Ref-1/APE and ERp57 in reductive activation of transcription factors. Free Radical Biology and Medicine 41 1113-1123. (https://doi.org/10.1016/j.freeradbiomed.2006.06.016)

Haupt Y, Maya R, Kazaz A \& Oren M 1997 Mdm2 promotes the rapid degradation of P53. Nature 387 296-299. (https://doi. org/10.1038/387296a0)

Hebert DN \& Molinari M 2007 In and out of the ER: protein folding, quality control, degradation, and related human diseases. Physiological Reviews 87 1377-1408. (https://doi.org/10.1152/physrev.00050.2006)

Hettinghouse A, Liu R \& Liu CJ 2018 Multifunctional molecule ERp57: from cancer to neurodegenerative diseases. Pharmacology and Therapeutics 181 34-48. (https://doi.org/10.1016/j.pharmthera.2017.07.011)
Huppertz B, Kadyrov M \& Kingdom JC 2006 Apoptosis and its role in the trophoblast. American Journal of Obstetrics and Gynecology 195 29-39. (https://doi.org/10.1016/j.ajog.2005.07.039)

Ishihara N, Matsuo H, Murakoshi H, Laoag-Fernandez JB, Samoto T \& Maruo T 2002 Increased apoptosis in the syncytiotrophoblast in human term placentas complicated by either preeclampsia or intrauterine growth retardation. American Journal of Obstetrics and Gynecology 186 158-166. (https://doi.org/10.1067/mob.2002.119176)

Krynetskaia NF, Phadke MS, Jadhav SH \& Krynetskiy EY 2009 Chromatinassociated proteins HMGB1/2 and PDIA3 trigger cellular response to chemotherapy-induced DNA damage. Molecular Cancer Therapeutics 8 864-872. (https://doi.org/10.1158/1535-7163.MCT-08-0695)

Kubbutat MHG, Jones SN \& Vousden KH 1997 Regulation of p53 stability by Mdm2. Nature 387 299-303. (https://doi.org/10.1038/387299a0)

Lahav G, Rosenfeld N, Sigal A, Geva-Zatorsky N, Levine AJ, Elowitz MB \& Alon U 2004 Dynamics of the p53-Mdm2 feedback loop in individual cells. Nature Genetics 36 147-150. (https://doi.org/10.1038/ng1293)

Longtine MS, Chen B, Odibo AO, Zhong Y \& Nelson DM 2012 Villous trophoblast apoptosis is elevated and restricted to cytotrophoblasts in pregnancies complicated by preeclampsia, IUGR, or preeclampsia with IUGR. Placenta 33 352-359. (https://doi.org/10.1016/j. placenta.2012.01.017)

Luongo de Matos L \& Trufelli DC 2010 Immunohistochemistry as an important tool in biomarkers detection and clinical practice MGLd matos, Maria, and AdS Pinhal. Biomarker Insights 5 9-20. (https://doi. org/10.4137/bmi.s2185)

Mo HQ, Tian FJ, Li X, Zhang J, Ma XL, Zeng WH, Lin Y \& Zhang Y 2019 ANXA7 regulates trophoblast proliferation and apoptosis in preeclampsia. American Journal of Reproductive Immunology 82 e13183. (https://doi.org/10.1111/aji.13183)

Mutze K, Vierkotten S, Milosevic J, Eickelberg O \& Konigshoff M 2015 Enolase 1 (ENO1) and protein disulfide-isomerase associated 3 (PDIA3) regulate $\mathrm{Wnt} /$ beta-catenin-driven trans-differentiation of murine alveolar epithelial cells. Disease Models and Mechanisms 8 877-890. (https:// doi.org/10.1242/dmm.019117)

Natalio G, Satoshi T, Frank M \& Günter HJ 2005 Impaired assembly of the major histocompatibility complex class I peptide-loading complex in mice deficient in the oxidoreductase ERp57. Nature Immunology 7 93-102. (https://doi.org/10.1038/ni1288)

Parakh S, Jagaraj CJ, Vidal M, Ragagnin AMG, Perri ER, Konopka A, Toth RP, Galper J, Blair IP, Thomas CJ et al. 2018 ERp57 is protective against mutant SOD1-induced cellular pathology in amyotrophic lateral sclerosis. Human Molecular Genetics 27 1311-1331. (https://doi. org/10.1093/hmg/ddy041)

Pressinotti NC, Klocker H, Schäfer G, Luu VD, Ruschhaupt M, Kuner R, Steiner E, Poustka A, Bartsch G \& Sültmann H 2009 Differential expression of apoptotic genes PDIA3 and MAP3K5 distinguishes between low- and high-risk prostate cancer. Molecular Cancer 8130. (https://doi.org/10.1186/1476-4598-8-130)

Sharp AN, Heazell AE, Crocker IP \& Mor G 2010 Placental apoptosis in health and disease. American Journal of Reproductive Immunology 64 159-169. (https://doi.org/10.1111/j.1600-0897.2010.00837.x)

Sharp AN, Heazell A, Baczyk D, Dunk CE, Lacey HA, Jones CJP, Perkins JE, Kingdom JCP et al. 2014 Preeclampsia is associated with alterations in the p53-pathway in villous trophoblast. PLoS One 9 e87621. (https://doi. org/10.1371/journal.pone.0087621)

Smith SC, Baker PN \& Symonds EM 1997 Increased placental apoptosis in intrauterine growth restriction. American Journal of Obstetrics and Gynecology 177 1395-1401. (https://doi.org/10.1016/s00029378(97)70081-4)

Smith SC, Leung TN, To KF \& Baker PN 2000 Apoptosis is a rare event in first-trimester placental tissue. American Journal of Obstetrics and Gynecology 183 697-699. (https://doi.org/10.1067/mob.2000.106555)

Sonderegger S, Yap J, Menkhorst E, Weston G, Stanton PG \& Dimitriadis E 2011 Interleukin (IL)11 mediates protein secretion and modification in human extravillous trophoblasts. Human Reproduction 26 2841-2849. (https://doi.org/10.1093/humrep/der259)

Tejera E, Bernardes J \& Rebelo I 2012 Preeclampsia: a bioinformatics approach through protein-protein interaction networks analysis. BMC Systems Biology 6 97. (https://doi.org/10.1186/1752-0509-6-97)

Tohda C, Urano T, Umezaki M, Nemere I \& Kuboyama T 2012 Diosgenin is an exogenous activator of 1,25D3-MARRS/Pdia3/ERp57 and improves 
Alzheimer's disease pathologies in 5XFAD mice. Scientific Reports 2 535. (https://doi.org/10.1038/srep00535)

Vogelstein B, Lane D \& Levine AJ 2000 Surfing the p53 network. Nature 408 307-310. (https://doi.org/10.1038/35042675)

Wang K, Li H, Chen R, Zhang Y, Sun XX, Huang W, Bian H \& Chen ZN 2017 Combination of CALR and PDIA3 is a potential prognostic biomarker for non-small cell lung cancer. Oncotarget 8 96945-96957. (https://doi.org/10.18632/oncotarget.18547)

Xu D, Perez RE, Rezaiekhaligh MH, Bourdi M \& Truog WE 2009 Knockdown of ERp57 increases BiP/GRP78 induction and protects against hyperoxia and tunicamycin-induced apoptosis. American Journal of Physiology: Lung Cellular and Molecular Physiology 297 L44-L51. (https://doi. org/10.1152/ajplung.90626.2008)

Yamamoto E, Uchida T, Abe H, Taka H, Fujimura T, Komiya K, Hara A, Ogihara T, Fujitani Y, Ueno T et al. 2014 Increased expression of ERp57/GRP58 is protective against pancreatic beta cell death caused by autophagic failure. Biochemical and Biophysical Research Communications 453 19-24. (https://doi.org/10.1016/j. bbrc.2014.09.040)

Yang Z, Liu J, Shi Q, Chao Y, Di Y, Sun J, Zhang J, Huang L, Guo H \& He C 2018 Expression of protein disulfide isomerase A3 precursor in colorectal cancer. OncoTargets and Therapy 11 4159-4166. (https://doi. org/10.2147/OTT.S154452)

Yuan G, Hua B, Yang Y, Xu L, Cai T, Sun N, Yan Z, Lu C \& Qian R 2017 The circadian gene clock regulates bone formation via PDIA3. Journal of Bone and Mineral Research 32 861-871. (https://doi.org/10.1002/ jbmr.3046)

Zou H, Wen C, Peng Z, Shao YY, Hu L, Li S, Li C \& Zhou HH 2018 P4HB and PDIA3 are associated with tumor progression and therapeutic outcome of diffuse gliomas. Oncology Reports 39 501-510. (https://doi. org/10.3892/or.2017.6134)

Received 20 March 2020

First decision 6 April 2020

Revised manuscript received 26 May 2020

Accepted 1 June 2020 